\title{
Bilateral pial synangiosis in a child with PHACE syndrome
}

\author{
Andrew S. Jack, MD, ${ }^{1}$ Michael M. Chow, MD, MPH, FRCSC, ${ }^{1}$ Loretta Fiorillo, MD, FRCPC, ${ }^{2}$ \\ Thea Chibuk, MD, FRCPC, ${ }^{3}$ Jerome Y. Yager, MD, FRCPC, ${ }^{4}$ and Vivek Mehta, MD, MSc, FRCSC ${ }^{1}$ \\ 1Division of Neurosurgery, Department of Surgery, and Divisions of ${ }^{2}$ Pediatric Dermatology, ${ }^{3}$ Pediatrics, and 4 Pediatric Neurology, \\ Department of Pediatrics, Stollery Children's Hospital, Edmonton, Alberta, Canada
}

\begin{abstract}
The acronym PHACE has been used to denote a constellation of abnormalities: posterior fossa anomalies, facial hemangiomas, arterial anomalies, cardiac anomalies, and eye abnormalities. Approximately $30 \%$ of patients with large facial hemangiomas have PHACE syndrome, with the vast majority having intracranial arteriopathy. Few reports characterize neurological deterioration from this intracranial arteriopathy, and even fewer report successful treatment thereof. The authors report on a case of a child with PHACE syndrome who presented with an ischemic stroke from a progressive intracranial arteriopathy and describe her successful treatment with bilateral pial synangiosis.
\end{abstract}

An 8-month old girl diagnosed with PHACE syndrome was found to have bilateral internal carotid artery stenosis. Although initially asymptomatic, a few months after diagnosis she suffered a right frontal and parietal stroke. MRI and cerebral angiography investigations demonstrated progressive intracranial arterial stenosis and occlusion. The patient then underwent indirect cerebral revascularization surgery. At 2-year follow-up, she exhibited clinical improvement with persistent speech and motor developmental delay. Follow-up MRI and cerebral angiography showed no new ischemic events and robust extensive vascular collateralization from surgery.

PHACE syndrome is an uncommon disease, and affected patients often have cerebral arteriopathy. Although the underlying natural history of cerebral arteriopathy in PHACE remains unclear, cerebral revascularization may represent a potential therapy for symptomatic patients.

http://thejns.org/doi/abs/10.3171/2015.5.PEDS1578

KEY WORDS PHACE; pial synangiosis; PHACES association; hemangioma; arterial ischemic syndrome; vascular anomalies; vascular disorders

$\mathrm{P}$ HACE is a rare neurocutaneous syndrome characterized by posterior fossa malformations, hemangiomas, arterial anomalies, aortic coarctation, cardiac defects, and eye abnormalities. Pascual-Castroviejo was the first to describe the relationship between facial hemangiomas and arteriopathies, ${ }^{23}$ which was subsequently expanded to its current form to also include ventral developmental defects such as a supraumbilical raphe and sternal clefting (PHACES). ${ }^{9}, 15,19,21$ The prevalence of PHACE syndrome among patients with large segmental facial hemangiomas is approximately $30 \%, 4,13$ and the most common extracutaneous finding in these patients is intracranial vascular abnormalities. ${ }^{4,7,13}$

Within PHACE syndrome a wide spectrum of vascular abnormalities exist, with stenosis and occlusion being among the most commonly described. To optimally man- age afflicted patients, insight into the natural history of this syndrome is required. Although large registries and cohort studies have recently been undertaken,,$^{13,14,22,26}$ the natural history of the arterial stenosis and occlusion associated with PHACE remains to be determined. Here, we describe a patient with PHACE syndrome presenting with symptomatic and progressive carotid artery stenosis treated via pial synangiosis with subsequent clinical improvement.

\section{Case Report}

History and Presentation

The patient had been born to nonconsanguineous healthy parents with an unremarkable family history. The gestation and delivery were uneventful. The patient was born at term, with a birth weight of $3.15 \mathrm{~kg}$, length of 48.9

ABBREVIATIONS $\mathrm{ACOA}=$ anterior communicating artery; $\mathrm{AIS}=$ arterial ischemic stroke; $\mathrm{AP}=$ anteroposterior; $\mathrm{CCA}=\mathrm{common}$ carotid artery; $\mathrm{ECA}=\mathrm{external}$ carotid artery; ICA = internal carotid artery; MCA = middle cerebral artery; MMD = moyamoya disease; $P C A=$ posterior cerebral artery; $P C O A=$ posterior communicating artery; STA = superficial temporal artery; VA = vertebral artery.

SUBMITTED February 4, 2015. ACCEPTED May 20, 2015

INCLUDE WHEN CITING Published online September 25, 2015; DOI: 10.3171/2015.5.PEDS1578. 
$\mathrm{cm}$, and APGAR scores of 9 and 10. Physical examination at the time revealed a healthy baby and was notable only for a pansystolic murmur. Cardiac ultrasonography revealed a moderate-sized ventricular septal defect, small atrial septal defect, and tricuspid regurgitation. A cardiology referral was made, resulting in conservative management of the cardiac defects, including clinical follow-up and repeat echocardiography.

At 5 months of age, the patient began developing multiple, large, segmental hemangiomas of the parotid glands bilaterally and the right maxillary and mandibular area (S2 and S3). A dermatological referral was made and MRI and MR angiography of the head were performed. The imaging revealed absence or occlusion of the left internal carotid artery (ICA) and stenosis of the right supraclinoid ICA (Fig. 1). The left middle cerebral artery (MCA) was being filled through the anterior communicating artery (ACoA) and, in retrograde flow, through the posterior communicating artery (PCoA). The right MCA territory also demonstrated collateral filling.

A diagnosis of PHACE syndrome was established. The patient was subsequently referred to the pediatric neurosurgical clinic regarding management of her abnormal cerebrovasculature. At this time the patient was at approximately the 50th percentile for both weight and length and had a normal head circumference. Her development was normal. On neurological examination no deficits were found, and expectant management with clinical follow-up and repeat MRI was ordered. As she had adequate collateral circulation, it was felt appropriate to commence propranolol treatment for her hemangiomas. This was started at $1 \mathrm{mg} / \mathrm{kg} / \mathrm{day}$ and later increased to $2 \mathrm{mg} / \mathrm{kg} / \mathrm{day}$. The latter dose proved effective and resulted in almost complete resolution of the hemangiomas by 11 months of age. However, prior to her next follow-up appointment the patient was brought into the pediatric emergency room after having a seizure. The patient was just under 18 months old at this time, in keeping with acute neurological deterioration documented in patients with PHACE syndrome. ${ }^{5}$ The patient was found to have a dense left hemiparesis and decreased level of consciousness. An emergent CT followed by MRI of the head revealed an infarct in the right frontal and parietal lobes (Fig. 2A and B). Cerebral angiography was also performed and showed a progressive high-grade stenosis of the right supraclinoid ICA and prominent right PCoA filling (Fig. 2C and D). Delayed cortical flow into the right MCA territory was noted. There was again absence of the left ICA, with the left common carotid artery (CCA) ending in the external carotid artery (ECA). The posterior circulation was unremarkable with the exception of a high-grade stenosis of the left PCoA at its junction with the anterior circulation (Fig. 2E). This stenosis resulted in a 3-second delay in filling between the left posterior and anterior circulation (concerning for significant left-side hypoperfusion).

\section{Surgical Treatment}

Due to the rarity of this disorder, a multidisciplinary meeting was convened regarding possible treatment options. Little literature exists describing in detail treatment options for progressive steno-occlusive cerebrovascular
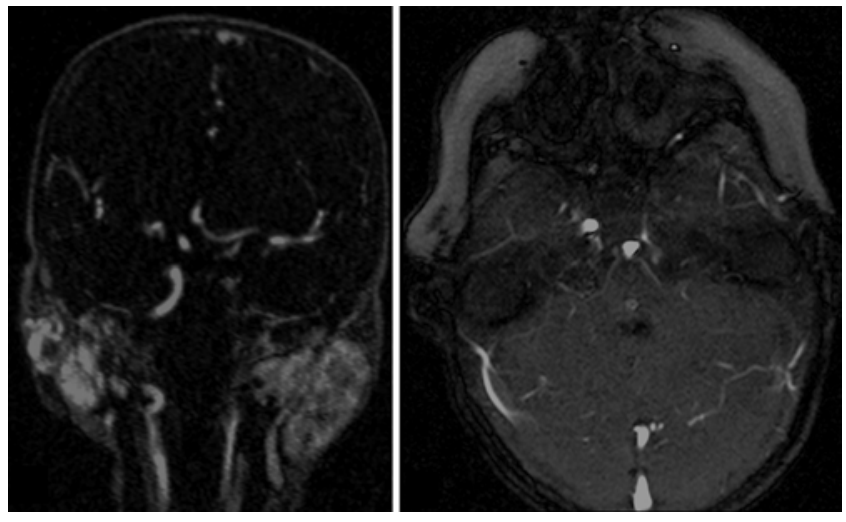

FIG. 1. Coronal (left) and axial (right) MR angiograms showing absence of the left ICA and filling of the left MCA and ACA territories as a result of cross-filling from the PCoA and ACoA.

disease in PHACE syndrome with, to our knowledge, only 1 prior report mentioning pial synangiosis. ${ }^{5}$ Thus personal communication was also sought from other centers (Dr. M. Scott and Dr. E. Smith, Boston Children's Hospital, and Dr. P. Dirks, The Hospital for Sick Children [SickKids], Toronto). A staged bilateral pial synangiosis was planned for 1 month poststroke, allowing the patient an initial period of recovery from the stroke. A left-sided pial synangiosis was first completed, followed by a right-sided approach a few weeks later. In both procedures, the superficial temporal artery (STA) was identified using Doppler ultrasonography. A linear incision was made over the frontoparietal area, and the STA was dissected under the microscope. The frontal branch was ligated anteriorly, and approximately $8.0 \mathrm{~cm}$ of the posterior branch of the STA was mobilized. The temporalis muscle was then incised and split, and a temporal craniotomy was performed. The dura and arachnoid mater were opened, and the STA was placed on the pia mater. The artery was secured to the pia using 4 interrupted, 10-0 Ethilon, sutures. An appropriately sized groove was fashioned in the bone flap to allow for the circuitous STA. The temporal bone flap was then replaced and secured with Vicryl, and Doppler ultrasonography was used to confirm patency of the graft both proximally and distally. Intraoperatively, cerebral oximetry was used by the anesthesiology team to help optimize sedation and hemodynamics.

\section{Postoperative Course}

The patient recovered from the surgeries and was referred to the pediatric brain injury and stroke rehabilitation program. Angiography performed 12 months postoperatively demonstrated extensive right and left MCA flow reconstitution via collateral circulation from the pial synangiosis (Fig. 3A and B). In this case, bilateral revascularization was completed successfully corresponding to angiographic Grade A collateralization as per the Matsushima grading scale..$^{18}$ This corresponds to an area greater than two-thirds of the MCA territory being perfused by the synangiosis.

The posterior circulation, however, was notable for new basilar artery terminus stenosis with posterior cere- 

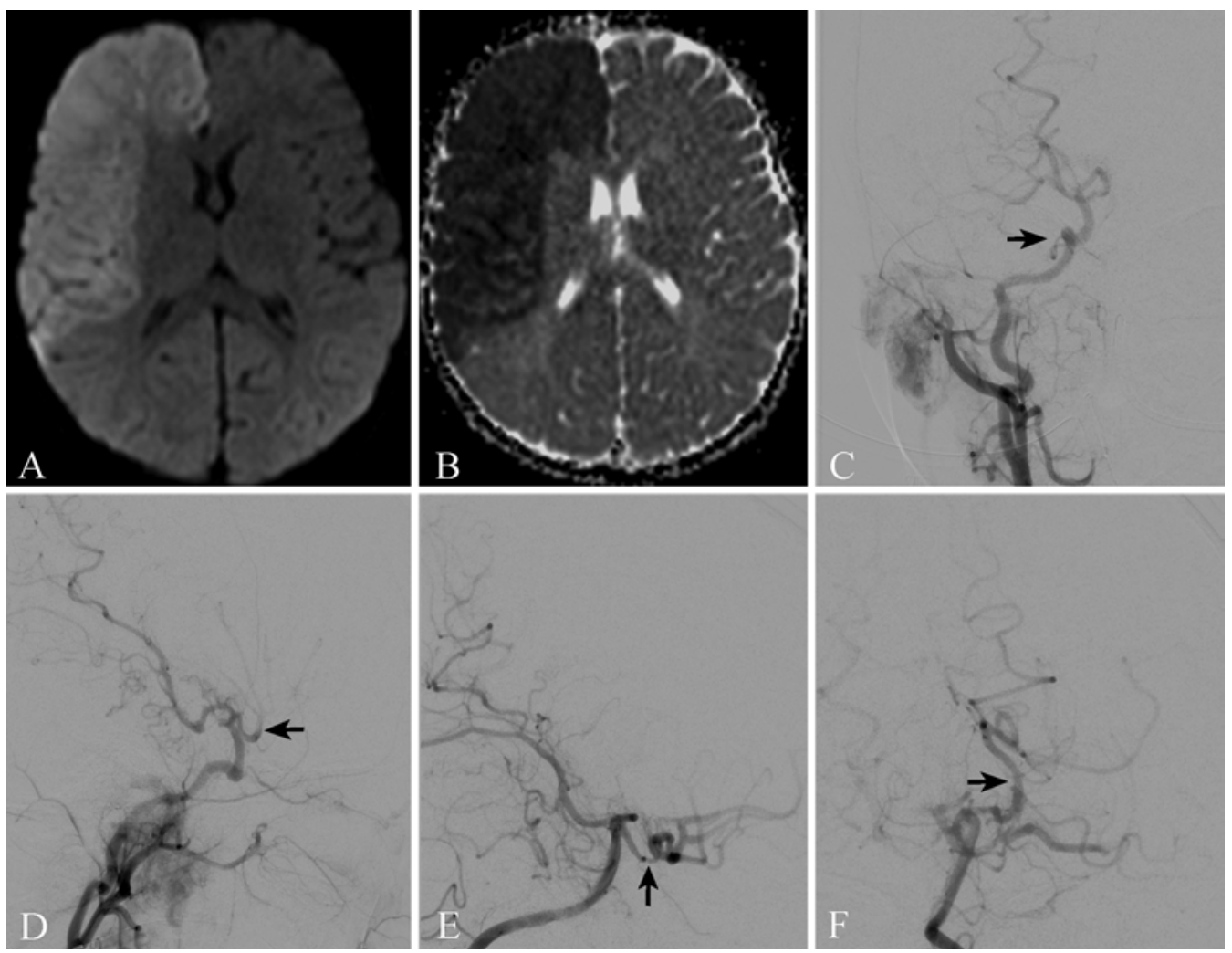

FIG. 2. A and B: Axial MR diffusion-weighted image (A) and apparent diffusion coefficient sequence (B) showing ischemic injury to the right frontal and parietal cortex. C: Anteroposterior (AP) angiographic projection of a right CCA injection demonstrating preferential filling of the PCA via the PCoA over MCA and ACA filling implying stenosis of the ICA distal to the PCoA origin. D: Lateral projection of the right CCA injection showing the stenosis of the supraclinoid ICA (arrow) and pial collaterals from the PCA. E: Lateral projection of a left vertebral artery (VA) injection demonstrating filling of the PCA and stenosis at the junction of the PCoA and anterior circulation (arrow). F: AP projection of a left VA injection demonstrating filling of the left PCA (arrow) and delayed filling of the anterior circulation through the PCOA.

bral artery (PCA) reconstitution via collateralization (Fig. $3 \mathrm{C})$. At the patient's most recent follow-up examination, 2 years after her stroke presentation she was doing well clinically, although moderate motor delays were present. Left-sided spasticity persisted from her previous stroke, but she was walking and running with little difficulty. An MRI examination performed at this follow-up visit demonstrated ongoing evolution of encephalomalacia in the right frontal area of the patient's previous stroke (Fig. 3D). In the absence of new neurological deficits, continued annual follow-up with MRA has been scheduled.

\section{Discussion}

Pascual-Castroviejo was the first to describe the relationship between facial hemangiomas and cerebral and facial arterial anomalies. ${ }^{23}$ Since that time, numerous inclusions and modifications have been proposed, resulting in what is now known as PHACE or PHACES syndrome. . $^{8,911,15,19}$ This condition poses somewhat of a diagnostic challenge as it can express itself as many different phenotypes. This has resulted in some confusion between "typical and atypical" cases, as well as between $\operatorname{PHACE}(\mathrm{S})$ and conditions such as Sturge-Weber syndrome. ${ }^{12,14,19,20}$ The relationship between conditions such as PHACE and the occurrence of sporadic hemangiomas is, at present, unclear. PHACE syndrome affects females far more often than males (9:1 female:male ratio), in contrast to sporadic hemangioma, which has a 3:1 female:male ratio. ${ }^{2}$ PHACE syndrome hemangiomas usually involve several cervicofacial segments; however, they are not confined to these segmental boundaries, unlike Sturge-Weber hemangiomas, which are typically confined to the $\mathrm{V} 1$ trigeminal region. The genetic and resultant phenotypic relationship to dermatological conditions such as Sturge-Weber and other steno-occlusive cerebrovascular diseases such as moyamoya disease (MMD) has yet to be determined. This is likely due to the idiopathic nature of both MMD and PHACE syndrome. Although the etiology is speculative, the broad spectrum of phenotypic abnormalities is explained in part by an early insult to neural crest cells and neural plate cells occurring before or early in vasculogenesis. ${ }^{2,14}$ However, as previously outlined, ${ }^{2}$ the precise insult, location, and timing thereof has yet to be elucidated. Despite the diagnostic and etiological challenges, consensus on criteria (major and minor) for definite and possible PHACE diagnosis has been reported. ${ }^{19}$ A diagnosis of PHACE syndrome requires having a facial hemangioma larger than $5 \mathrm{~cm}$ in diameter and 1 major criterion or 2 minor criteria. Possible examples of PHACE syndrome include: having a facial hemangio- 


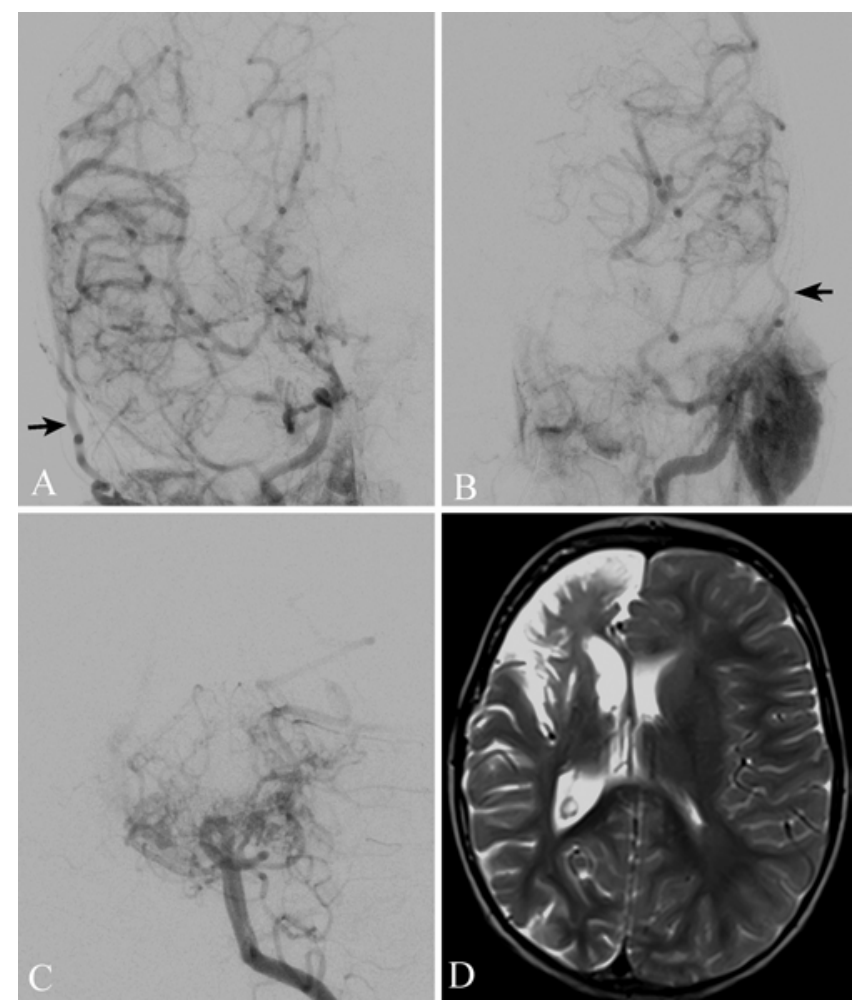

FIG. 3. A: Postoperative angiographic AP projection of a right CCA injection demonstrating extensive collateralization and filling of the anterior circulation territory via the ECA graft (arrow). B: Postoperative angiographic AP projection of a left CCA injection demonstrating increased collateralization and filling of the anterior circulation territory via the ECA graft (arrow). C: Postoperative angiographic lateral projection of a left VA injection showing progressive basilar terminus collateralization and decreased filling of the proximal left PCA. D: Postoperative axial T2-weighted MR image showing encephalomalacia of the right frontoparietal area.

ma larger than $5 \mathrm{~cm}$ in diameter and 1 minor criterion, a neck or upper torso hemangioma and 1 major criterion or 2 minor criteria, or simply having 2 major criteria abnormalities. A complete list of the major and minor criteria abnormalities has been previously reported elsewhere. ${ }^{19}$ Our patient met diagnostic criteria as she had a facial hemangioma larger than $5 \mathrm{~cm}$ as well as anomalies of her major cerebral arteries.

Although uncommon, PHACE syndrome is not a rare disorder and may represent the most common neurocutaneous vascular disorder. ${ }^{19,21,22}$ Several large registries and cohort studies have been reported outlining the prevalence and incidence of the disease's different abnormalities. However, little has been reported regarding the natural history of the syndrome. More specifically, few have reported on the natural history of the intracranial arteriopathy, which affects approximately $90 \%$ of PHACE patients. ${ }^{13}$ Although rates of intracranial structural and cerebrovascular anomalies vary among PHACE patients, these represent the most common extracutaneous abnormalities associated with the condition, occurring in $72 \%-94 \%$ of patients. ${ }^{13,14,19,22}$ The cerebrovascular anomalies found in PHACE syndrome are widely diverse, but they have been classified into 4 groups: dysplasia (including ectasia, looping, kinking, and/or fusiform dilation), narrowing (including developmental hypoplasia or agenesis and acquired stenosis or occlusion), vascular course or origin aberrancy, and persistent embryonic anastomoses. ${ }^{19}$ Despite characterization of these vascular anomalies, little is known regarding their natural history.

In previous studies, $50 \%$ of PHACE patients with intracranial arteriopathy were found to become symptomatic from progressive vasculopathy. ${ }^{5,22}$ Burrows et al. first reported the progression of congenital cerebrovascular anomalies in PHACE with serial neuroimaging. ${ }^{5}$ In their study, neuroimaging had initially been completed documenting the baseline cerebrovascular abnormalities. Subsequent follow-up imaging was completed for 4 of the 8 patients experiencing acute neurological symptoms between 9 and 18 months of age. Three of these patients had developed occlusion or stenosis of the terminal ICA and/ or proximal ACA and MCA. These patients also demonstrated collateralization similar to that seen in MMD. ${ }^{5}$ Several other potential cases have been reported documenting arterial ischemic stroke (AIS), seizures, and progressive neurological deficits in PHACE patients, ${ }^{1-3,9,17,22,23}$ but incomplete information prevents definitive diagnosis. Furthermore, retrospective studies have reported progressive arterial occlusions and stenosis occurring in $20.9 \%$ and $18.3 \%$ of PHACE patients, respectively, with progression being shown to occur as quickly as over the course of 1 month on follow-up neurovascular imaging., ${ }^{4}, 14$ It has been proposed that serial neuroimaging in patients with PHACE syndrome is justified given the progressive nature of the arteriopathy in some of these patients. ${ }^{22}$ Moreover, neurosurgical revascularization procedures such as pial synangiosis could potentially reduce AIS-related morbidity and mortality. ${ }^{5}$

The dilemma of how to approach a patient with an asymptomatic arteriopathy is not unique. Lin et al. studied 83 children with aysmptomatic moyamoya arteriopathy. ${ }^{16}$ Within their patient cohort, 54\% of the patients demonstrated radiographic progression within a mean of 5.4 years of follow-up. The authors evaluated both radiographic and clinical progression. Children with sickle cell anemia and neurofibromatosis Type 1 were at higher risk of progression. Guidelines regarding treatment via revascularization surgery have been published for MMD; patients for whom revascularization surgery is recommended include those with ongoing ischemic events and/or cerebral blood flow and perfusion reserve..$^{29} \mathrm{In}$ contrast to the situation with respect to MMD, a relative lack of literature documenting the natural history of arteriopathy in PHACE patients results in difficulty developing such guidelines for the care of asymptomatic patients.

Another pertinent issue pertains to the natural history and management of a child with PHACES syndrome who has only a unilateral vascular problem. Smith and Scott studied 33 children who underwent surgical treatment for unilateral moyamoya disease. Within this subgroup, onethird of the children developed a progressive arteriopathy on the contralateral side. Risk factors in that study included any imaging abnormality on the contralateral side at the time of presentation, previous cranial irradiation, 
congenital cardiac anomaly, Asian ancestry, and familial moyamoya syndrome. ${ }^{28}$ Furthermore, following patients after cerebral revascularization and identifying the extent of collateralization often proves difficult. Repeat angiography is often required, and there can be difficulty in accurately qualifying changes in the extent of revascularization. For example, the Matsushima grading scale ${ }^{18}$ can be used, but this scale is based largely on MCA territory collateralization and is inappropriate for evaluating ACA and/or PCA vascular territory revascularization.

Many techniques have been described for surgical revascularization in children. $6,24,25,27,30,31$ Most of the surgical literature distinguishes between direct and indirect revascularization techniques. Questions within pediatric neurosurgery still exist on which is the best technique. It is unclear how quickly the indirect technique works to revascularize the brain. Previous groups have reported a series of children that have been treated with either indirect or direct procedures. In one reported case series, a direct superficial temporal artery to middle cerebral artery anastomosis is considered in children over the age of 4 when the donor vessel is at least $0.7 \mathrm{~mm}$ in diameter. ${ }^{31}$

Many surgical treatment options have been outlined for conditions such as moyamoya syndrome, ${ }^{6,24,27,30}$ and the durability of pial synangiosis in preventing stroke for children with moyamoya syndrome has been previously described..$^{25}$ It is unclear with PHACE syndrome if the extracranial donor vessel will continue to function and protect the brain against further cerebral ischemia. As a result, the dilemma as to the best treatment for intracranial vasculopathy is largely unresolved. Little evidence exists regarding medical therapy, such as intravenous interferon or steroids, for the arteriopathies, mainly due to the unknown mechanism behind these conditions. The other difficult question with respect to treatment of these patients is related to knowing which subset will benefit from revascularization, as well as the timing of the procedure. It is difficult to determine the most appropriate timing for surgical revascularization for PHACE patients. MMD guidelines regarding timing of surgical intervention suggest minimizing the time between diagnosis and treatment, although delays to increase the safety of the procedure are warranted, and unstable disease has been shown to increase perioperative morbidity. ${ }^{10,29}$ Furthermore, revascularization surgery may prove futile if the extracranial vasculature undergoes the same progressive stenosis and occlusion. Moreover, there is little evidence documenting concrete risk factors for neurological complications related to abnormal cerebrovasculature in PHACE patients. ${ }^{22}$ Our patient demonstrated progressive intracranial arteriopathy with subsequent neurological deterioration. Therefore, intracranial revascularization was performed, and clinical and radiological improvement was documented. Further investigation is required to identify those PHACE patients at risk for neurological compromise due to intracranial vasculopathy so that they may be treated prior to deterioration.

\section{Conclusions}

PHACE syndrome consists of a broad spectrum of dis- ease manifestations, and it is often difficult to diagnose as well as treat. Progressive intracranial vasculopathy and the timing of intervention represent significant challenges. We present the case of a patient with delayed neurological deterioration in the face of progressive intracranial arteriopathy, successfully treated via bilateral pial synangiosis. Further study is required to elucidate which PHACE patients are at risk for complications from cerebral vascular abnormalities and their optimal treatment.

\section{References}

1. Aeby A, Guerrini R, David P, Rodesch G, Raybaud C, Van Bogaert P: Facial hemangioma and cerebral corticovascular dysplasia: a syndrome associated with epilepsy. Neurology 60:1030-1032, 2003

2. Bhattacharya JJ, Luo CB, Alvarez H, Rodesch G, Pongpech S, Lasjaunias PL: PHACES syndrome: a review of eight previously unreported cases with late arterial occlusions. Neuroradiology 46:227-233, 2004

3. Billson VR, Gillam GL: An unusual case of Sturge-Weber syndrome. Pathology 16:462-465, 1984

4. Bracken J, Robinson I, Snow A, Watson R, Irvine AD, Rea $\mathrm{D}$, et al: PHACE syndrome: MRI of intracerebral vascular anomalies and clinical findings in a series of 12 patients. Pediatr Radiol 41:1129-1138, 2011

5. Burrows PE, Robertson RL, Mulliken JB, Beardsley DS, Chaloupka JC, Ezekowitz RA, et al: Cerebral vasculopathy and neurologic sequelae in infants with cervicofacial hemangioma: report of eight patients. Radiology 207:601-607, 1998

6. Dauser RC, Tuite GF, McCluggage CW: Dural inversion procedure for moyamoya disease. Technical note. J Neurosurg 86:719-723, 1997

7. Drolet BA, Dohil M, Golomb MR, Wells R, Murowski L, Tamburro J, et al: Early stroke and cerebral vasculopathy in children with facial hemangiomas and PHACE association. Pediatrics 117:959-964, 2006

8. Frieden IJ, Haggstrom AN, Drolet BA, Mancini AJ, Friedlander SF, Boon L, et al: Infantile hemangiomas: current knowledge, future directions. Proceedings of a research workshop on infantile hemangiomas, April 7-9, 2005, Bethesda, Maryland, USA. Pediatr Dermatol 22:383-406, 2005

9. Frieden IJ, Reese V, Cohen D: PHACE syndrome. The association of posterior fossa brain malformations, hemangiomas, arterial anomalies, coarctation of the aorta and cardiac defects, and eye abnormalities. Arch Dermatol 132:307-311, 1996

10. Funaki T, Takahashi JC, Takagi Y, Kikuchi T, Yoshida K, Mitsuhara T, et al: Unstable moyamoya disease: clinical features and impact on perioperative ischemic complications. J Neurosurg 122:400-407, 2015

11. Goh WH, Lo R: A new 3C syndrome: cerebellar hypoplasia, cavernous haemangioma and coarctation of the aorta. Dev Med Child Neurol 35:637-641, 1993

12. Grosso S, De Cosmo L, Bonifazi E, Galluzzi P, Farnetani MA, Loffredo P, et al: Facial hemangioma and malformation of the cortical development: a broadening of the PHACE spectrum or a new entity? Am J Med Genet A 124A:192195,2004

13. Haggstrom AN, Garzon MC, Baselga E, Chamlin SL, Frieden IJ, Holland K, et al: Risk for PHACE syndrome in infants with large facial hemangiomas. Pediatrics 126:e418e426, 2010

14. Heyer GL, Dowling MM, Licht DJ, Tay SK, Morel K, Garzon MC, et al: The cerebral vasculopathy of PHACES syndrome. Stroke 39:308-316, 2008 
15. James PA, McGaughran J: Complete overlap of PHACE syndrome and sternal malformation-vascular dysplasia association. Am J Med Genet 110:78-84, 2002

16. Lin N, Baird L, Koss M, Kopecky KE, Gone E, Ullrich NJ, et al: Discovery of asymptomatic moyamoya arteriopathy in pediatric syndromic populations: radiographic and clinical progression. Neurosurg Focus 31(6):E6, 2011

17. Luo CB, Lasjaunias P, Teng MM, Chang FC, Lirng JF, Chang CY: Cervico-cerebrovascular anomalies in children with PHACE syndrome. J Formos Med Assoc 102:379-386, 2003

18. Matsushima Y, Inaba Y: Moyamoya disease in children and its surgical treatment. Introduction of a new surgical procedure and its follow-up angiograms. Childs Brain 11:155170, 1984

19. Metry D, Heyer G, Hess C, Garzon M, Haggstrom A, Frommelt P, et al: Consensus Statement on Diagnostic Criteria for PHACE Syndrome. Pediatrics 124:1447-1456, 2009

20. Metry DW, Dowd CF, Barkovich AJ, Frieden IJ: The many faces of PHACE syndrome. J Pediatr 139:117-123, 2001

21. Metry DW, Garzon MC, Drolet BA, Frommelt P, Haggstrom A, Hall J, et al: PHACE syndrome: current knowledge, future directions. Pediatr Dermatol 26:381-398, 2009

22. Metry DW, Haggstrom AN, Drolet BA, Baselga E, Chamlin S, Garzon M, et al: A prospective study of PHACE syndrome in infantile hemangiomas: demographic features, clinical findings, and complications. Am J Med Genet A 140:975986, 2006

23. Pascual-Castroviejo I, Viaño J, Moreno F, Palencia R, Martínez Fernandez V, Pascual-Pascual SI, et al: Hemangiomas of the head, neck, and chest with associated vascular and brain anomalies: a complex neurocutaneous syndrome. AJNR Am J Neuroradiol 17:461-471, 1996

24. Patel NN, Mangano FT, Klimo P Jr: Indirect revascularization techniques for treating moyamoya disease. Neurosurg Clin N Am 21:553-563, 2010

25. Scott RM, Smith JL, Robertson RL, Madsen JR, Soriano SG, Rockoff MA: Long-term outcome in children with moyamoya syndrome after cranial revascularization by pial synangiosis. J Neurosurg 100 (2 Suppl Pediatrics):142-149, 2004

26. Siegel DH, Tefft KA, Kelly T, Johnson C, Metry D, Burrows $\mathrm{P}$, et al: Stroke in children with posterior fossa brain malfor- mations, hemangiomas, arterial anomalies, coarctation of the aorta and cardiac defects, and eye abnormalities (PHACE) syndrome: a systematic review of the literature. Stroke 43:1672-1674, 2012

27. Smith ER, McClain CD, Heeney M, Scott RM: Pial synangiosis in patients with moyamoya syndrome and sickle cell anemia: perioperative management and surgical outcome. Neurosurg Focus 26(4):E10, 2009

28. Smith ER, Scott RM: Progression of disease in unilateral moyamoya syndrome. Neurosurg Focus 24(2):E17, 2008

29. Smith ER, Scott RM: Spontaneous occlusion of the circle of Willis in children: pediatric moyamoya summary with proposed evidence-based practice guidelines. A review. J Neurosurg Pediatr 9:353-360, 2012

30. Smith ER, Scott RM: Surgical management of moyamoya syndrome. Skull Base 15:15-26, 2005

31. Veeravagu A, Guzman R, Patil CG, Hou LC, Lee M, Steinberg GK: Moyamoya disease in pediatric patients: outcomes of neurosurgical interventions. Neurosurg Focus 24(2):E16, 2008

\section{Disclosure}

The authors report no conflict of interest concerning the materials or methods used in this study or the findings specified in this paper.

\section{Author Contributions}

Conception and design: Jack, Chow, Mehta. Acquisition of data: Jack, Chow, Mehta. Analysis and interpretation of data: all authors. Drafting the article: Jack, Fiorillo, Chibuk, Mehta. Critically revising the article: all authors. Reviewed submitted version of manuscript: Jack, Chow, Fiorillo, Chibuk, Mehta. Approved the final version of the manuscript on behalf of all authors: Jack. Study supervision: Mehta.

\section{Correspondence}

Andrew S. Jack, Division of Neurosurgery, Department of Surgery, 2D1.02 Mackenzie Centre, University of Alberta Hospital, Edmonton, AB T6G 2B7, Canada. email: asjack@ualberta.ca. 